Homology, Homotopy and Applications, vol.12(1), 2010, pp.45-60

\title{
ON THE WU INVARIANTS FOR IMMERSIONS OF A GRAPH INTO THE PLANE
}

\author{
RYO NIKKUNI
}

(communicated by Alexander Mishchenko)

\begin{abstract}
We give an explicit calculation of the Wu invariants for immersions of a finite graph into the plane and classify all generic immersions of a graph into the plane up to regular homotopy by the $\mathrm{Wu}$ invariant. This result is a generalization of the fact that two plane curves are regularly homotopic if and only if they have the same rotation number.
\end{abstract}

\section{Introduction}

Throughout this paper we work in the piecewise linear category. In $[\mathbf{6}, \mathbf{7}], \mathrm{Wu}$ defined an isotopy invariant of embeddings and immersions of polyhedra into the Euclidean space in terms of the cohomology of deleted product spaces. In case of embeddings, this invariant classifies all embeddings of a graph into $\mathbb{R}^{3}$ up to spatial graph-homology (Taniyama [4]). But as far as the author knows, little is known about an application of this invariant in case of immersions. Our purpose in this paper is to give an explicit calculation of Wu's invariant of immersions of a graph into $\mathbb{R}^{2}$ and apply it to a geometric classification.

Let $G$ be a finite, connected and simple graph which has at least one edge. We denote the set of all vertices (resp. edges) of $G$ by $V(G)$ (resp. $E(G)$ ). If the terminal vertices of an edge $e$ of $G$ are $u$ and $v$, then we denote $e=(u, v)=(v, u)$. We denote the number of edges incident to a vertex $v$ by $\operatorname{deg} v$. Note that $G$ has a structure of a finite 1-dimensional simplicial complex. We regard $G$ as a topological space by considering its geometric realization, namely $G$ is a compact and connected 1-dimensional polyhedron. In this situation, each of the vertices and the edges of $G$ can be regarded as a subset of $G$. We call a continuous map $f: G \rightarrow \mathbb{R}^{2}$ a plane immersion of $G$ if there exists an open covering $\left\{U_{\nu}\right\}$ of $G$ such that $\left.f\right|_{U_{\nu}}$ is an embedding for any $\nu$. A plane immersion $f$ of $G$ is said to be generic if all of its multipoints are transversal double points away from vertices. We say that two plane immersions $f$ and $g$ of $G$ are regularly homotopic if there exists a homotopy $F: G \times[0,1] \rightarrow \mathbb{R}^{2}$ from $f$ to $g$ and an open covering $\left\{U_{\nu}\right\}$ of $G$ such that $\left.f_{t}\right|_{U_{\nu}}$ is an embedding for any $\nu$ and for any $t \in[0,1]$, where $f_{t}$ is a continuous map from $G$ to $\mathbb{R}^{2}$ defined by

Received July 8, 2009, revised September 30, 2009; published on February 24, 2010.

2000 Mathematics Subject Classification: 57Q35, 57M15.

Key words and phrases: immersion, graph, Wu invariant.

This article is available at http://intlpress.com/HHA/v12/n1/a5

Copyright (C) 2010, International Press. Permission to copy for private use granted. 
$f_{t}(x)=F(x, t)$ for any $x \in G$. Note that regular homotopy defines an equivalence relation on plane immersions of a graph. ${ }^{1}$

We give the precise definition of the Wu invariant $\mathcal{R}(f)$ of a plane immersion $f$ of a graph in the next section and also give an explicit calculation of $\mathcal{R}(f)$ in Section 3. It can be calculated as a first cohomology class of a subspace of the symmetric deleted product of the graph, which is called the symmetric tube of the graph. Moreover, we have the following classification theorem.

Theorem 1.1. Let $f$ and $g$ be two generic plane immersions of a graph $G$. Then the following are equivalent:

(1) $f$ and $g$ are regularly homotopic.

(2) $f$ and $g$ are transformed into each other by the local moves as illustrated in Figure 1(1), (2), (3) and ambient isotopies.

(3) $\mathcal{R}(f)=\mathcal{R}(g)$.

(1)

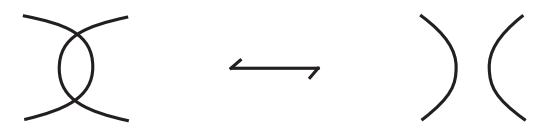

(2)

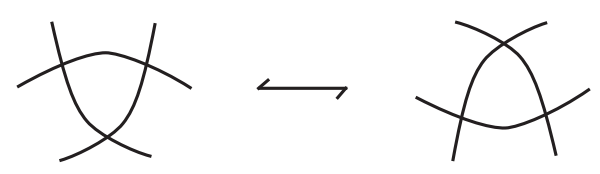

(3)

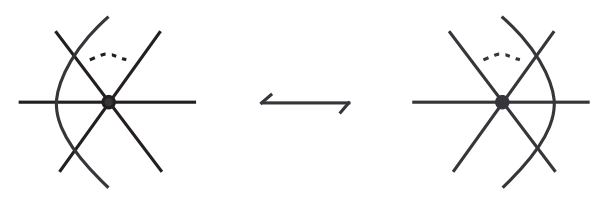

Figure 1.

Let $K_{m}$ be the complete graph on $m$ vertices for a positive integer $m$, namely $V\left(K_{m}\right)=\left\{v_{1}, v_{2}, \ldots, v_{m}\right\}$ and $E\left(K_{m}\right)=\left\{\left(v_{i}, v_{j}\right)(1 \leqslant i<j \leqslant m)\right\}$. A plane immersion $f$ of $K_{3}$ is called a plane curve. By Theorem 1.1, we have the following corollary.

Corollary 1.2. Let $f$ and $g$ be two generic plane curves. Then the following are equivalent:

(1) $f$ and $g$ are regularly homotopic.

(2) $f$ and $g$ are transformed into each other by the local moves as illustrated in Figure 1(1), (2) and ambient isotopies.

\footnotetext{
${ }^{1}$ This equivalence relation was introduced in $[\mathbf{6}]$ by the name of local isotopy.
} 
(3) $\mathcal{R}(f)=\mathcal{R}(g)$.

We prove Theorem 1.1 in Section 4. As we will see in Example 3.11, $\mathcal{R}(f)$ of a plane curve $f$ coincides with the rotation number [5] of $f$. (In this paper we consider the orientation on $\mathbb{R}^{2}$ with positive rotation numbers in the counterclockwise direction.) Thus Corollary 1.2 coincides with the regular homotopy classification of plane curves by Whitney-Graustein's theorem [5] and Kauffman's combinatorial interpretation [2]. We remark here that recently Permyakov gives a simple combinatorial interpretation of $\mathcal{R}(f)$ and shows a theorem which corresponds to Theorem $1.1[\mathbf{3}]$.

\section{Acknowledgements}

The author is most grateful to Professors Tohl Asoh and Tsutomu Yasui for their invaluable comments.

\section{Wu invariant}

In this section we give the definition of the Wu invariant of a plane immersion of a graph $G$. We refer the reader to $[\mathbf{6}]$ for the general case. Let $X$ be a topological space. For the embedding $\tilde{d}: X \rightarrow X \times X$ defined by $\tilde{d}(x)=(x, x)$, we call $\widetilde{X}^{*}=$ $(X \times X) \backslash \tilde{d}(X)$ the deleted product of $X$. A map $\sigma(x, y)=(y, x)$ gives a free $\mathbb{Z}_{2^{-}}$ action on $\widetilde{X}^{*}$. We call $X^{*}=\widetilde{X}^{*} / \mathbb{Z}_{2}$ the symmetric deleted product of $X$. We denote the image of $\tilde{d}(X)$ by the natural projection from $X \times X$ to $(X \times X) / \mathbb{Z}_{2}$ by $d(X)$. Let $U$ be a neighborhood of $\tilde{d}(X)$ in $X \times X$. Then $\widetilde{U}^{*}=U \backslash \tilde{d}(X)$ is called a deleted neighborhood of $\tilde{d}(X)$ in $\widetilde{X}^{*}$. A deleted neighborhood $\widetilde{U}^{*}$ is said to be $\sigma$-invariant if $\sigma(U)=U$. Then we call $U^{*}=\widetilde{U}^{*} / \mathbb{Z}_{2}$ a symmetric deleted neighborhood of $d(X)$ in $X^{*}$.

For a graph $G$, let $\left\{U_{\lambda}^{*}\right\}$ be the set of all symmetric deleted neighborhoods of $d(G)$ in $G^{*}$. Then $\left\{U_{\lambda}^{*}, \prec\right\}$ forms an oriented set by $U_{\lambda}^{*} \prec U_{\mu}^{*}$ if $U_{\lambda}^{*} \supset U_{\mu}^{*}$. For this oriented set, $\left\{H^{1}\left(U_{\lambda}^{*} ; \mathbb{Z}\right), i_{\lambda}^{\mu *}\right\}$ forms an inductive system of modules, where $H^{1}(\cdot ; \mathbb{Z})$ denotes the integral first cohomology group and

$$
i_{\lambda}^{\mu^{*}}: H^{1}\left(U_{\lambda}^{*} ; \mathbb{Z}\right) \rightarrow H^{1}\left(U_{\mu}^{*} ; \mathbb{Z}\right)
$$

is a homomorphism induced by the inclusion. Then we denote the inductive limit $\stackrel{\lim }{\longrightarrow} H^{1}\left(U_{\lambda}^{*} ; \mathbb{Z}\right)$ by $R(G)$. We note that we have the following natural homomorphism

$$
i_{\lambda}^{*}: H^{1}\left(U_{\lambda}^{*} ; \mathbb{Z}\right) \longrightarrow R(G)
$$

for any symmetric deleted neighborhood $U_{\lambda}^{*}$ of $d(G)$ in $G^{*}$.

Let $f: G \rightarrow \mathbb{R}^{2}$ be a plane immersion. Namely there exists an open covering $\mathcal{U}=$ $\left\{U_{\nu}\right\}$ of $G$ such that $\left.f\right|_{U_{\nu}}$ is an embedding for any $\nu$. Then the set

$$
\widetilde{W}_{\mathcal{U}}=\left\{\left(x_{1}, x_{2}\right) \in \widetilde{G}^{*} \mid \text { there exists a } U_{\nu} \text { such that } x_{1}, x_{2} \in U_{\nu}\right\}
$$

forms a $\sigma$-invariant deleted neighborhood of $\tilde{d}(G)$ in $\widetilde{G}^{*}$ and a continuous map $\bar{f}: W_{\mathcal{U}} \rightarrow\left(\mathbb{R}^{2}\right)^{*}$ is defined by $\bar{f}\left[x_{1}, x_{2}\right]=\left[f\left(x_{1}\right), f\left(x_{2}\right)\right]$. On the other hand, it is well 
known that a continuous map $r:\left(\widetilde{\mathbb{R}}^{2}\right)^{*} \rightarrow \mathbb{S}^{1}$ defined by

$$
r\left(y_{1}, y_{2}\right)=\left(y_{1}-y_{2}\right) /\left\|y_{1}-y_{2}\right\|
$$

is a $\sigma$-equivariant strong deformation retract and $r:\left(\mathbb{R}^{2}\right)^{*} \rightarrow \mathbb{S}^{1} / \mathbb{Z}_{2} \approx \mathbb{S}^{1}$ is also a strong deformation retract, where $\mathbb{S}^{1} / \mathbb{Z}_{2}$ denotes the quotient space of $\mathbb{S}^{1}$ by identifying the antipodal points. Let $\Sigma$ be a generator of $H^{1}\left(\mathbb{S}^{1} ; \mathbb{Z}\right) \cong \mathbb{Z}$. Then the image of $\Sigma$ by the composition

$$
H^{1}\left(\mathbb{S}^{1} ; \mathbb{Z}\right) \stackrel{r^{*}}{\cong} H^{1}\left(\mathbb{R}^{2^{*}} ; \mathbb{Z}\right) \stackrel{\bar{f}^{*}}{\longrightarrow} H^{1}\left(W_{\mathcal{U}} ; \mathbb{Z}\right) \stackrel{i_{\mathcal{U}}^{*}}{\longrightarrow} R(G)
$$

is denoted by $\mathcal{R}(f)$, where $i_{\mathcal{U}}^{*}$ is the natural homomorphim of $(2.1)$ for $W_{\mathcal{U}}$. We call $\mathcal{R}(f)$ a $W u$ invariant ${ }^{2}$ of $f$. We remark here that the definition above is independent of the choice of $\mathcal{U}$.

Proposition $2.1([6])$. $\mathcal{R}(f)$ is a regular homotopy invariant.

Proof. Let $f$ and $g$ be two regularly homotopic plane immersions of $G$. Namely there exists a homotopy $F: G \times[0,1] \rightarrow \mathbb{R}^{2}$ from $f$ to $g$ and an open covering $\left\{U_{\nu}\right\}$ of $G$ such that $\left.f_{t}\right|_{U_{\nu}}$ is an embedding for any $\nu$ and for any $t \in[0,1]$, where $f_{t}(x)=F(x, t)$ for $x \in G$. Then we can define a homotopy $F_{\mathcal{U}}: W_{\mathcal{U}} \times[0,1] \longrightarrow\left(\mathbb{R}^{2}\right)^{*}$ from $\bar{f}$ to $\bar{g}$ by $F_{\mathcal{U}}\left(\left[x_{1}, x_{2}\right], t\right)=\left[f_{t}\left(x_{1}\right), f_{t}\left(x_{2}\right)\right]$. Thus we have that $\mathcal{R}(f)=i_{\mathcal{U}}^{*} \bar{f}^{*} \bar{r}^{*}(\Sigma)=i_{\mathcal{U}}^{*} \bar{g}^{*} \bar{r}^{*}(\Sigma)=$ $\mathcal{R}(g)$. This completes the proof.

\section{Symmetric tube of a graph}

A precise method to calculate $R(G)$ is provided in [6]. Let $X$ and $Y$ be two topological spaces and $M=X \cup(X \times Y \times[0,1]) \cup Y$ the disjoint union. Let us consider a quotient space by identifying $(x, y, 0) \in X \times Y \times[0,1]$ with $x \in X$ and $(x, y, 1) \in$ $X \times Y \times[0,1]$ with $y \in Y$. We call the quotient space a join of $X$ and $Y$ and denote it by $X \circ Y$. We set $[X, Y]^{(0)}=\{[x, y, 1 / 2] \in X \circ Y \mid x \in X, y \in Y\}$. For example, the join $v$ oe of a vertex $v$ and an edge $e$ is homeomorphic to a 2-simplex, and $[v, e]^{(0)}$ is homeomorphic to a 1-simplex. The following is a special case of what is called a canonical cellular decomposition of the product space of $X[\mathbf{1}, \mathbf{6}]$.

Proposition 3.1. Let $G$ be a graph. Then $G \times G$ is decomposed into the following cells:

(1) $\tilde{d}(s)$ for $s \in V(G)$ or $E(G)$.

(2) $s_{1} \times s_{2}$ for $s_{i} \in V(G)$ or $E(G)(i=1,2)$ and $s_{1} \cap s_{2}=\emptyset$.

(3) $\tilde{d}(s) \circ\left(s_{1} \times s_{2}\right)$ for $s, s_{1}, s_{2} \in V(G)$ or $E(G), s_{1} \cap s_{2}=\emptyset$ and $s \cup s_{i}$ is contained in a vertex or an edge of $G(i=1,2)$.

In particular, the cellular complex which consists of all cells $\left[s, s_{1} \times s_{2}\right]^{(0)}$ for simplices $s, s_{1}$ and $s_{2}$ of Proposition 3.1(3) is called a tube of $G$ and is denoted by $\widetilde{G}^{(0)}$. Clearly $\widetilde{G}^{(0)}$ is $\sigma$-invariant in $\widetilde{G}^{*}$. We call $\widetilde{G}^{(0)} / \mathbb{Z}_{2}$ a symmetric tube of $G$ and denote it by $G^{(0)}$. We denote $\left[s, s_{1} \times s_{2}\right]^{(0)} / \mathbb{Z}_{2}$ by $\left[s, s_{1} * s_{2}\right]$, and we note that $\left[s, s_{1} * s_{2}\right]=\left[s, s_{2} * s_{1}\right]$.

\footnotetext{
${ }^{2}$ This invariant was introduced in $[6]$ by the name of local isotopy class and denoted by $\Lambda_{f}(G)$.
} 
Example 3.2. Let $K_{3}$ be the complete graph on three vertices as illustrated in Figure 2. The figure on the right side in Figure 2 illustrates the canonical cellular decomposition of $K_{3} \times K_{3}$ in the sense of Proposition 3.1 as an expanded diagram of the 2-dimensional torus. The dotted thick parts and black thick parts represent the cells of Proposition 3.1(1) and (2), respectively. The gray thick parts represent $\widetilde{K}_{3}^{(0)}$. Thus we can see that $K_{3}^{(0)}$ is homeomorphic to the circle.
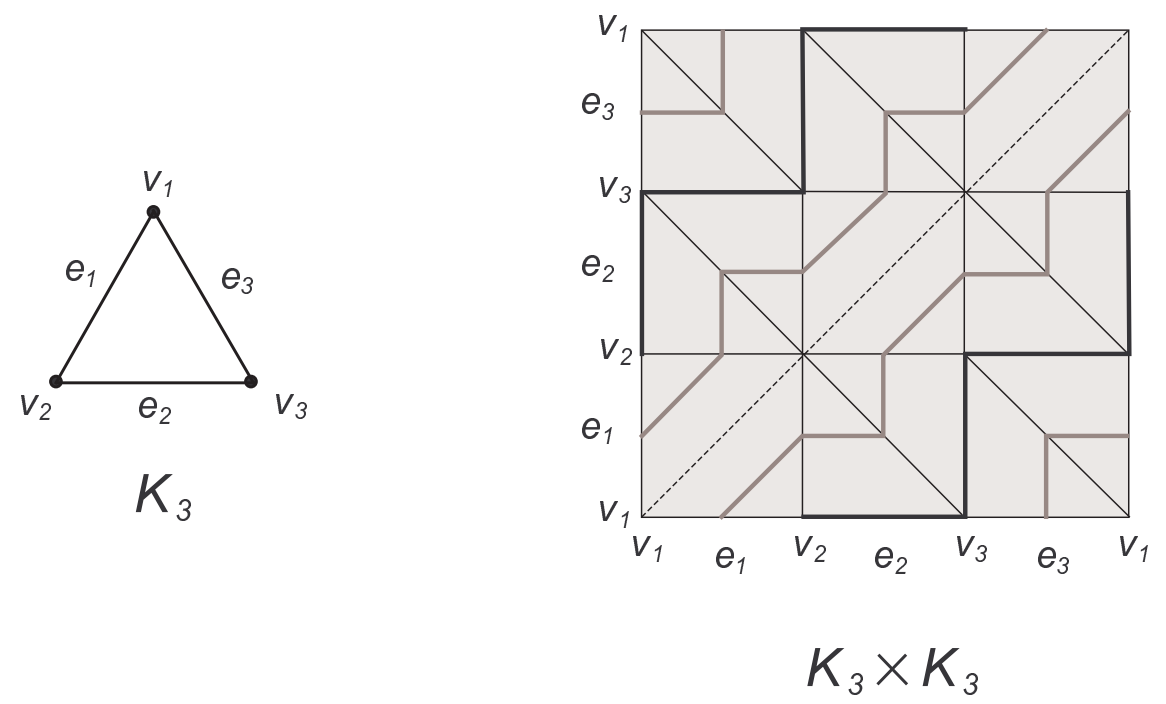

Figure 2.

Let $P\left(\widetilde{G}^{*}\right)$ be the cellular complex which consists of all cells $s_{1} \times s_{2}$ for simplices $s_{1}, s_{2}$ of Proposition 3.1(2). Clearly $P\left(\widetilde{G}^{*}\right)$ is also $\sigma$-invariant in $\widetilde{G}^{*}$. We denote $P\left(\widetilde{G}^{*}\right) / \mathbb{Z}_{2}$ by $P\left(G^{*}\right)$. We note that $G^{*} \backslash P\left(G^{*}\right)$ is a symmetric deleted neighborhood of $d(G)$ in $G^{*}$. It is known that

$$
i_{G^{*} \backslash P\left(G^{*}\right)}^{*}: H^{1}\left(G^{*} \backslash P\left(G^{*}\right) ; \mathbb{Z}\right) \stackrel{\cong}{\longrightarrow} R(G),
$$

where $i_{G^{*} \backslash P\left(G^{*}\right)}$ is the natural homomorphism of (2.1) for $G^{*} \backslash P\left(G^{*}\right)$, and there exists a deformation retract $j: G^{*} \backslash P\left(G^{*}\right) \rightarrow G^{(0)}[\mathbf{6}]$. Therefore we have the following.

Theorem $3.3([6]) \cdot i_{G^{(0)}}^{*}=i_{G^{*} \backslash P\left(G^{*}\right)}^{*} j^{*}: H^{1}\left(G^{(0)} ; \mathbb{Z}\right) \stackrel{\cong}{\longrightarrow} R(G)$.

Thus the calculation of $H^{1}\left(G^{(0)} ; \mathbb{Z}\right)$ provides a precise method to calculate $R(G)$. To calculate $H^{1}\left(G^{(0)} ; \mathbb{Z}\right)$, we investigate the structure of $G^{(0)}$ directly. We set $V(G)=$ $\left\{v_{1}, v_{2}, \ldots, v_{m}\right\}$ and $E(G)=\left\{e_{1}, e_{2}, \ldots, e_{n}\right\}$. We choose a fixed orientation on each 
edge of $G$. We put

$$
\begin{aligned}
Z_{s t}^{s}=Z_{t s}^{s}=\left[v_{s}, v_{s} * v_{t}\right] & \text { for }\left(v_{s}, v_{t}\right) \in E(G) \\
W_{s t}^{u}=W_{t s}^{u}=\left[v_{u}, v_{s} * v_{t}\right] & \text { for }\left(v_{u}, v_{s}\right),\left(v_{u}, v_{t}\right) \in E(G), v_{s} \neq v_{t}, \\
X_{s t}^{i}=X_{t s}^{i}=\left[e_{i}, v_{s} * v_{t}\right] & \text { for } e_{i}=\left(v_{s}, v_{t}\right) \in E(G),
\end{aligned}
$$

and

$$
Y_{t i}^{s}=\left[v_{s}, v_{t} * e_{i}\right] \quad \text { for }\left(v_{s}, v_{t}\right), e_{i} \in E(G),\left(v_{s}, v_{t}\right) \neq e_{i} \text { and } v_{s} \subset e_{i} .
$$

Note that $Z_{s t}^{s}$ and $W_{s t}^{u}$ are 0 -dimensional simplices of $G^{(0)}$, and $X_{s t}^{i}$ and $Y_{t i}^{s}$ are 1-dimensional simplices of $G^{(0)}$. An orientation of $X_{s t}^{i}$ is induced by $e_{i}$, and an orientation of $Y_{t i}^{s}$ is induced by $e_{i}$. We can consider $Z_{s t}^{s}$ and $W_{s t}^{u}$ as 0 -chains in $C_{0}\left(G^{(0)} ; \mathbb{Z}\right)$ and $X_{s t}^{i}$ and $Y_{t i}^{s}$ as 1 -chains in $C_{1}\left(G^{(0)} ; \mathbb{Z}\right)$. The dual cochain of $Z_{s t}^{s}, W_{s t}^{u}, X_{s t}^{i}$ and $Y_{t i}^{s}$ are denoted by $Z_{s}^{s t}, W_{u}^{s t}, X_{i}^{s t}$ and $Y_{s}^{t i}$, respectively. It is not difficult to see the following.

Proposition 3.4. For a graph $G$, a cell of symmetric tube $G^{(0)}$ is one of $Z_{s t}^{s}$, $W_{s t}^{u}$, $X_{s t}^{i}$ and $Y_{\text {ti }}^{s}$ as above. Therefore $G^{(0)}$ is also a graph.

For example, let $S_{n}$ be a graph as illustrated in Figure 3. By enumerating vertices and edges of $S_{n}^{(0)}$ and observing the connection between them directly, we have the following.

Lemma 3.5. The symmetric tube $S_{n}^{(0)}$ of $S_{n}$ is a graph as follows:

(1) $V\left(S_{n}^{(0)}\right)=\left\{Z_{n+1, i}^{i}\right.$ and $\left.Z_{n+1, i}^{n+1}(i=1,2, \ldots, n), W_{j k}^{n+1}(1 \leqslant j<k \leqslant n)\right\}$.

(2) $E\left(S_{n}^{(0)}\right)=\left\{X_{n+1, i}^{i}(i=1,2, \ldots, n), Y_{j k}^{n+1}\right.$ and $\left.Y_{k j}^{n+1}(1 \leqslant j<k \leqslant n)\right\}$.

(3) $X_{n+1, i}^{i}=\left(Z_{n+1, i}^{n+1}, Z_{n+1, i}^{i}\right)(i=1,2, \ldots, n), Y_{j k}^{n+1}=\left(Z_{n+1, j}^{n+1}, W_{j k}^{n+1}\right)$ and $Y_{k j}^{n+1}=$ $\left(Z_{n+1, k}^{n+1}, W_{j k}^{n+1}\right)(1 \leqslant j<k \leqslant n)$.

(4) $\operatorname{deg} Z_{n+1, i}^{i}=1, \operatorname{deg} Z_{n+1, i}^{n+1}=n(i=1,2, \ldots, n)$ and $\operatorname{deg} W_{j k}^{n+1}=2(1 \leqslant j<k \leqslant n)$.

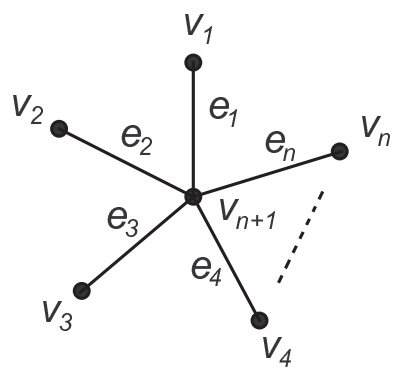

Figure 3. 
Example 3.6. Figure 4 illustrates the symmetric tube $S_{n}^{(0)}$ of $S_{n}$ for $n=1,2$ and 3 . We can see that the subgraph $H$ of $S_{n}^{(0)}$ defined by

$$
\begin{aligned}
& V(H)=\left\{Z_{n+1, i}^{n+1}(i=1,2, \ldots, n), W_{j k}^{n+1}(1 \leqslant j<k \leqslant n)\right\}, \\
& E(H)=\left\{Y_{j k}^{n+1}, Y_{k j}^{n+1}(1 \leqslant j<k \leqslant n)\right\}
\end{aligned}
$$

is homeomorphic to $K_{n}$. Precisely speaking, $H$ is isomorphic to the graph which is obtained from $K_{n}$ by subdividing each edge of $K_{n}$ once.

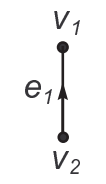

$S_{1}$

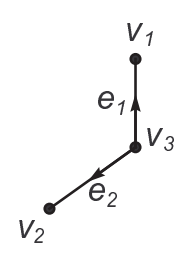

$S_{2}$

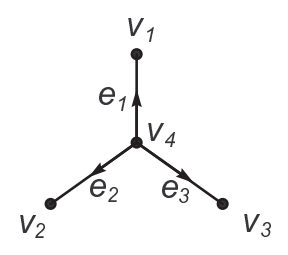

$S_{3}$

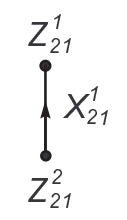

$S_{1}^{(0)}$
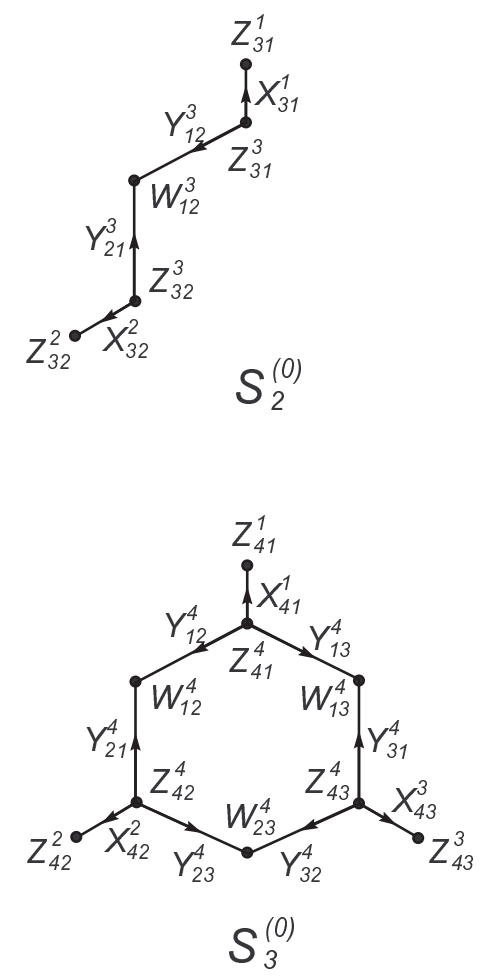

Figure 4.

By Lemma 3.5 and Example 3.6, we can see that the symmetric tube $G^{(0)}$ of a graph 
$G$ is obtained from $G$ by "substituting" $K_{d}$ for each vertex $v_{s}$ of $G$ as follows, where $d=\operatorname{deg} v_{s}$. Also see Examples 3.11 and 3.13.

Lemma 3.7. Let $G$ be a graph. For a vertex $v_{s}$ of $G$, let $v_{s_{1}}, v_{s_{2}}, \ldots, v_{s_{d}}$ be all vertices connected to $v_{s}$ so that $e_{i_{l}}=\left(v_{s}, v_{s_{l}}\right)\left(l=1,2, \ldots, d, 1 \leqslant i_{j}<i_{k} \leqslant d\right.$ for $\left.j<k\right)$. Then $G^{(0)}$ is obtained from $G$ by replacing each $v_{s}$ with the graph $H_{s}$, which is homeomorphic to $K_{d}$ defined by

$$
\begin{aligned}
& V\left(H_{s}\right)=\left\{Z_{s s_{l}}^{s}(i=1,2, \ldots, d), W_{s_{j} s_{k}}^{s}(1 \leqslant j<k \leqslant d)\right\}, \\
& E\left(H_{s}\right)=\left\{Y_{s_{j} i_{k}}^{s}=\left(Z_{s s_{j}}^{s}, W_{s_{j} s_{k}}^{s}\right), Y_{s_{k} i_{j}}^{s}=\left(Z_{s s_{k}}^{s}, W_{s_{j} s_{k}}^{s}\right)(1 \leqslant j<k \leqslant d)\right\},
\end{aligned}
$$

and by replacing $e_{i_{l}}$ with $X_{s s_{l}}^{i_{l}}=\left(Z_{s s_{l}}^{s_{l}}, Z_{s s_{l}}^{s}\right)(l=1,2, \ldots, d)$.

By the universal coefficient theorem, it is sufficient to know $H_{1}\left(G^{(0)} ; \mathbb{Z}\right)$ to calculate $H^{1}\left(G^{(0)} ; \mathbb{Z}\right)$. So in the following we construct a spanning tree of $G^{(0)}$. First we define a subgraph $T_{S_{n}^{(0)}}$ of $S_{n}^{(0)}$ as follows. We set $V\left(T_{S_{n}^{(0)}}\right)=V\left(S_{n}^{(0)}\right)$ and

$$
\begin{aligned}
E\left(T_{S_{n}^{(0)}}\right)= & \left\{X_{n+1, i}^{i}(i=1,2, \ldots, n), Y_{n j}^{n+1}(j=1,2, \ldots, n-1),\right. \\
& \left.Y_{t n}^{n+1}(t=1,2, \ldots, n-1), Y_{k l}^{n+1}(1 \leqslant k<l \leqslant n-1)\right\} .
\end{aligned}
$$

We note that

$$
\left.E\left(S_{n}^{(0)}\right) \backslash E\left(T_{S_{n}^{(0)}}\right)=\left\{Y_{l k}^{n+1}(1 \leqslant k<l \leqslant n-1)\right)\right\} .
$$

Then we easily have the following.

Lemma 3.8. A subgraph $T_{S_{n}^{(0)}}$ is a spanning tree of $S_{n}^{(0)}$.

Now we construct a spanning tree of $G^{(0)}$ on the outcome of Lemma 3.8. For $v_{s} \in V(G)$, let $\operatorname{st}\left(v_{s}\right)$ be a subgraph of $G$ consisting of $v_{s}$ and all edges incident to $v_{s}$. Let $v_{s_{1}}, v_{s_{2}}, \ldots, v_{s_{d}}$ be all vertices connected to $v_{s}$ so that

$$
e_{i_{l}}=\left(v_{s}, v_{s_{l}}\right)\left(l=1,2, \ldots, d, 1 \leqslant i_{j}<i_{k} \leqslant d \text { for } j<k\right),
$$

where $d=\operatorname{deg} v_{s}$. We construct a spanning tree $T_{\mathrm{st}\left(v_{s}\right)}$ of $\operatorname{st}\left(v_{s}\right)$ in the same way as $S_{n}^{(0)}$. Namely, $V\left(T_{\mathrm{st}\left(v_{s}\right)}\right)=V\left(\operatorname{st}\left(v_{s}\right)\right)$ and

$$
\begin{aligned}
E\left(T_{\mathrm{st}\left(v_{s}\right)}\right)= & \left\{X_{s s_{l}}^{i_{l}}(l=1,2, \ldots, d), Y_{s_{d} i_{j}}^{s}(j=1,2, \ldots, d-1),\right. \\
& \left.Y_{s_{j} i_{d}}^{s}(j=1,2, \ldots, d-1), Y_{s_{j} i_{k}}^{s}(1 \leqslant j<k \leqslant d-1)\right\} .
\end{aligned}
$$

Let $T_{G}$ be a spanning tree of $G$. We define a subgraph $T_{G^{(0)}}$ of $G^{(0)}$ by $V\left(T_{G^{(0)}}\right)=$ $V\left(G^{(0)}\right)$ and

$$
\begin{aligned}
E\left(T_{G^{(0)}}\right)= & \left\{X_{j_{1} j_{2}}^{j} \mid e_{j}=\left(v_{j_{1}}, v_{j_{2}}\right) \in E\left(T_{G}\right)\right\} \\
& \cup \bigcup_{v_{s} \in V(G)}\left(E\left(T_{\mathrm{st}\left(v_{s}\right)}\right) \backslash\left\{X_{s s_{l}}^{i_{l}}(l=1,2, \ldots, d)\right\}\right) .
\end{aligned}
$$

Then we have the following.

Lemma 3.9. A subgraph $T_{G^{(0)}}$ is a spanning tree of $G^{(0)}$. 
We note that

$$
E\left(\operatorname{st}\left(v_{s}\right)\right) \backslash E\left(T_{\text {st }\left(v_{s}\right)}\right)=\left\{Y_{s_{j} i_{k}}^{s}(1 \leqslant j<k \leqslant d-1)\right\}
$$

for $v_{s} \in V(G)$ and this set is empty for $d=1,2$. Therefore we have that

$$
\begin{aligned}
E\left(G^{(0)}\right) \backslash E\left(T_{G^{(0)}}\right)= & \left\{X_{j_{1} j_{2}}^{j} \mid e_{j}=\left(v_{j_{1}}, v_{j_{2}}\right) \in E(G) \backslash E\left(T_{G}\right)\right\} \\
& \cup \bigcup_{\substack{v_{s} \in V(G) \\
\operatorname{deg} v_{s} \geqslant 3}}\left\{Y_{s_{j} i_{k}}^{s}(1 \leqslant j<k \leqslant d-1)\right\} .
\end{aligned}
$$

Thus we can determine a structure of $H^{1}\left(G^{(0)} ; \mathbb{Z}\right)$ completely as follows.

Theorem 3.10.

(1) $H^{1}\left(G^{(0)} ; \mathbb{Z}\right) \cong \bigoplus_{\substack{e_{j} \in E(G) \backslash E\left(T_{G}\right) \\ e_{j}=\left(v_{j_{1}}, v_{j_{2}}\right)}}\left\langle X_{j}^{j_{1} j_{2}}\right\rangle \oplus \bigoplus_{\substack{v_{s} \in V(G) \\ \operatorname{deg} v_{s} \geqslant 3}}\left(\bigoplus_{1 \leqslant j<k \leqslant d-1}\left\langle Y_{s}^{s_{j} i_{k}}\right\rangle\right)$,

(2) $\operatorname{rank} H^{1}\left(G^{(0)} ; \mathbb{Z}\right)=1-2 n+\frac{1}{2} \sum_{s=1}^{m}\left(\operatorname{deg} v_{s}\right)^{2}$.

Proof. (1) is clear. We show (2). We have that

$$
\begin{aligned}
\operatorname{rank} H^{1}\left(G^{(0)} ; \mathbb{Z}\right) & =\operatorname{rank} H^{1}(G ; \mathbb{Z})+\sum_{s=1}^{m} \frac{1}{2}\left(\operatorname{deg} v_{s}-1\right)\left(\operatorname{deg} v_{s}-2\right) \\
& =n-m+1+\frac{1}{2} \sum_{s=1}^{m}\left\{\left(\operatorname{deg} v_{s}\right)^{2}-3 \operatorname{deg} v_{s}+2\right\} \\
& =n-m+1+\frac{1}{2} \sum_{s=1}^{m}\left(\operatorname{deg} v_{s}\right)^{2}-\frac{3}{2} \sum_{s=1}^{m} \operatorname{deg} v_{s}+m \\
& =n+1+\frac{1}{2} \sum_{s=1}^{m}\left(\operatorname{deg} v_{s}\right)^{2}-3 n \\
& =1-2 n+\frac{1}{2} \sum_{s=1}^{m}\left(\operatorname{deg} v_{s}\right)^{2} .
\end{aligned}
$$

This completes the proof.

Example 3.11. Let $K_{3}$ be the complete graph on three vertices as illustrated in the left side of Figure 5. As we saw in Example 3.2, the symmetric tube $K_{3}^{(0)}$ is a graph as illustrated in Figure 5. For a spanning tree $T_{K_{3}}=e_{1} \cup e_{2}$ of $K_{3}$, by Theorem 3.10 we have that $H^{1}\left(K_{3}^{(0)} ; \mathbb{Z}\right)=\left\langle X_{1}^{12}\right\rangle \cong \mathbb{Z}$. We note that if $[x, y, 1 / 2] \in K_{3}^{(0)}$ rotates once around the one in the direction induced by the orientation of $X_{12}^{1}$ then the non-ordered pair $(x, y)$ rotates once around $K_{3}$; see Figure 6 . Here the initial and terminal points of a vector in Figure 6 correspond to $x$ and $y$, respectively. This shows that $\mathcal{R}(f)$ of a plane curve $f$ coincides with the rotation number of $f$.

Example 3.12. For $S_{3}$ and its symmetric tube $S_{3}^{(0)}$ as illustrated in Figure 4, by Theorem 3.10 we have that $H^{1}\left(S_{3}^{(0)} ; \mathbb{Z}\right)=\left\langle Y_{4}^{21}\right\rangle \cong \mathbb{Z}$. We note that if $[x, y, 1 / 2] \in S_{3}^{(0)}$ 

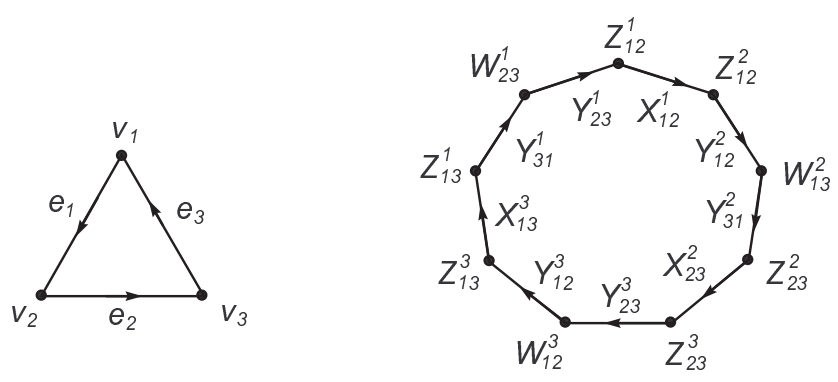

$K_{3}$

$K_{3}^{(0)}$

Figure 5.

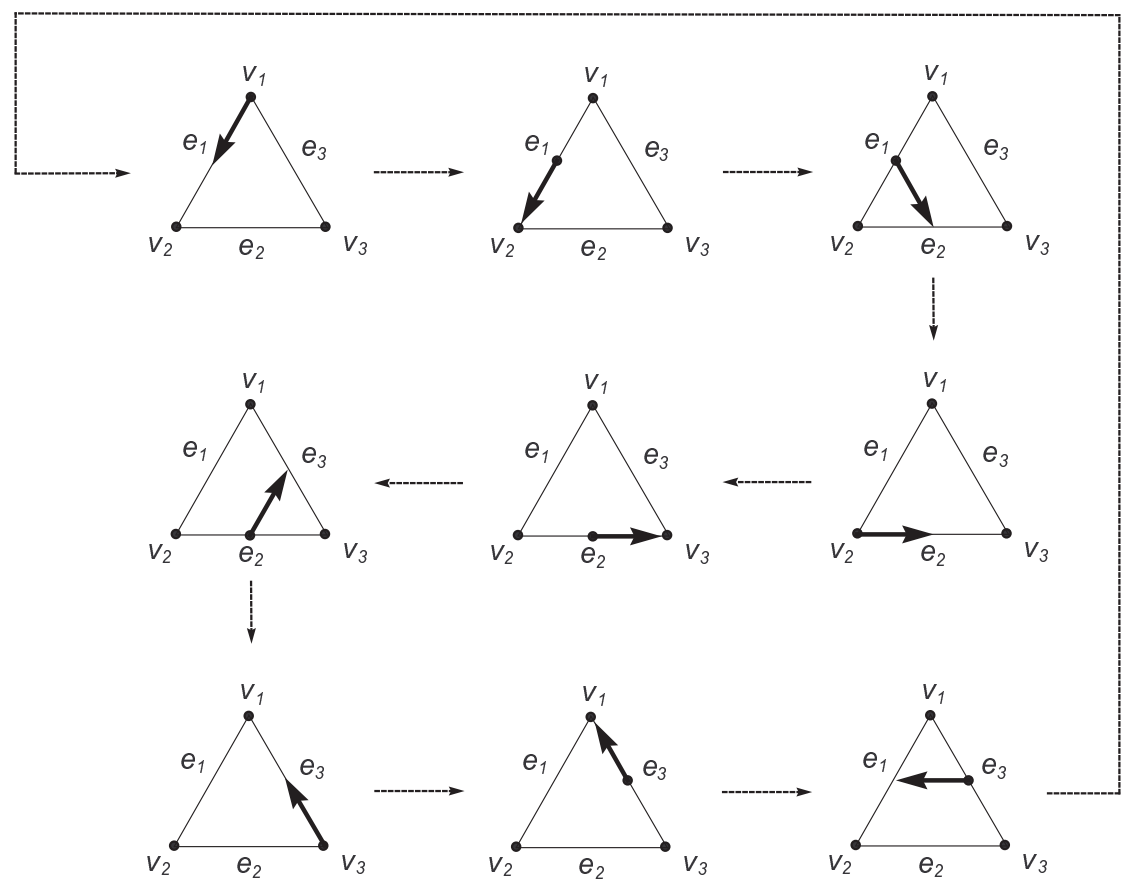

Figure 6 . 
rotates once around the cycle represented by $Y_{21}^{4}$ in the direction induced by the orientation of the one then the non-ordered pair $(x, y)$ rotates once around $v_{4}$; see Figure 7 . Here the initial and terminal points of a vector in Figure 7 correspond to $x$ and $y$, respectively.

Let $f$ be a generic plane immersion of $S_{3}$. Then there exists a neighbourhood $U$ of $v_{4}$ such that $\left.f\right|_{U}$ is an embedding. We can see that $\mathcal{R}(f)=1$ if $\left.f\right|_{U}\left(e_{1} \cap U\right),\left.f\right|_{U}\left(e_{2} \cap U\right)$ and $\left.f\right|_{U}\left(e_{3} \cap U\right)$ are embedded in $\mathbb{R}^{2}$ as illustrated in Figure $8(1)$, and $\mathcal{R}(f)=-1$ if $\left.f\right|_{U}\left(e_{1} \cap U\right),\left.f\right|_{U}\left(e_{2} \cap U\right)$ and $\left.f\right|_{U}\left(e_{3} \cap U\right)$ are embedded in $\mathbb{R}^{2}$ as illustrated in Figure 8(2).

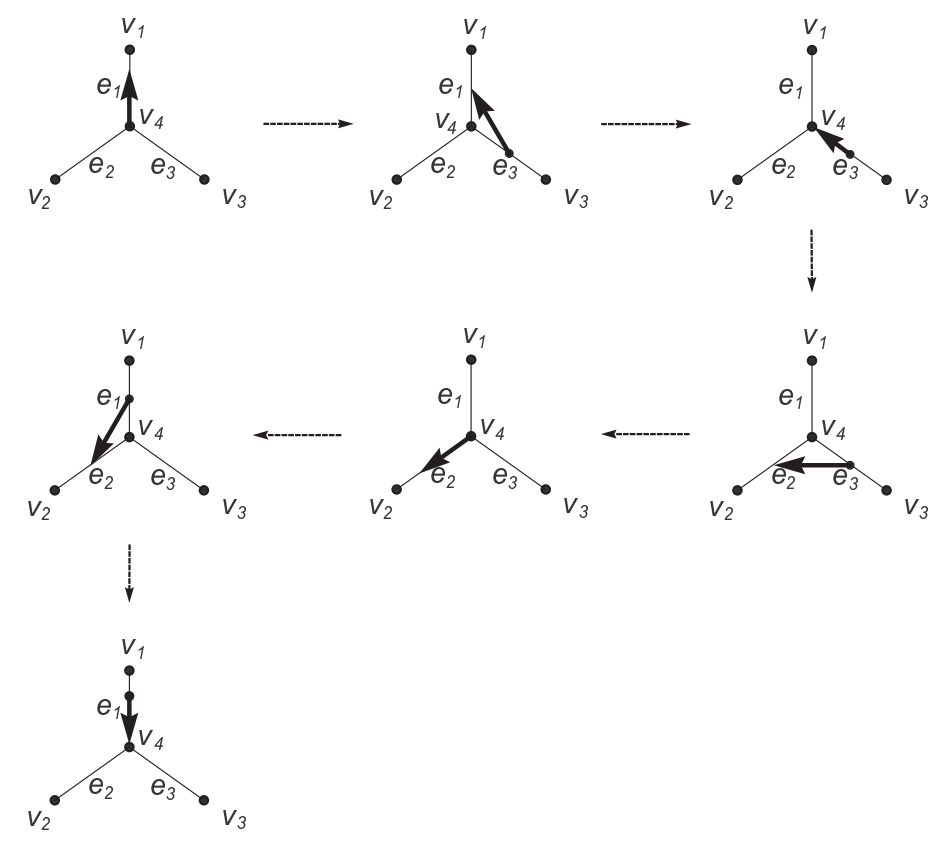

Figure 7 .

Example 3.13. Let $K_{4}$ be the complete graph on four vertices and $f, g$ and $h$ three generic plane immersions of $K_{4}$ as illustrated in Figure 9. Then the symmetric tube of $K_{4}$ is a graph as illustrated in Figure 10. For a spanning tree $T_{K_{4}}=e_{1} \cup e_{2} \cup e_{3}$ of $K_{4}$, by Theorem 3.10 we have that

$$
H^{1}\left(K_{4}^{(0)} ; \mathbb{Z}\right)=\left\langle X_{4}^{23}, X_{5}^{24}, X_{6}^{34}, Y_{1}^{31}, Y_{2}^{31}, Y_{3}^{22}, Y_{4}^{23}\right\rangle \cong \underbrace{\mathbb{Z} \oplus \mathbb{Z} \oplus \cdots \oplus \mathbb{Z}}_{\text {seven times }} .
$$

By calculating on the outcome of Examples 3.11 and 3.12, we have that

$$
\begin{aligned}
& \mathcal{R}(f)=(-1,1,-1,1,1,1,1), \\
& \mathcal{R}(g)=(1,-1,1,1,-1,1,-1)
\end{aligned}
$$




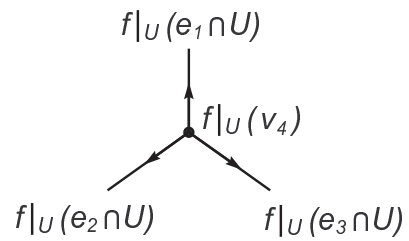

(1)

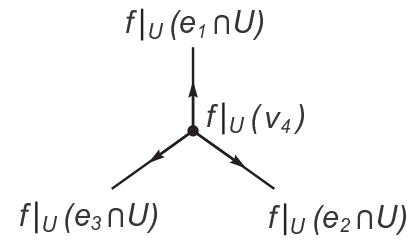

(2)

Figure 8.

and

$$
\mathcal{R}(h)=(0,0,0,1,1,-1,1) .
$$

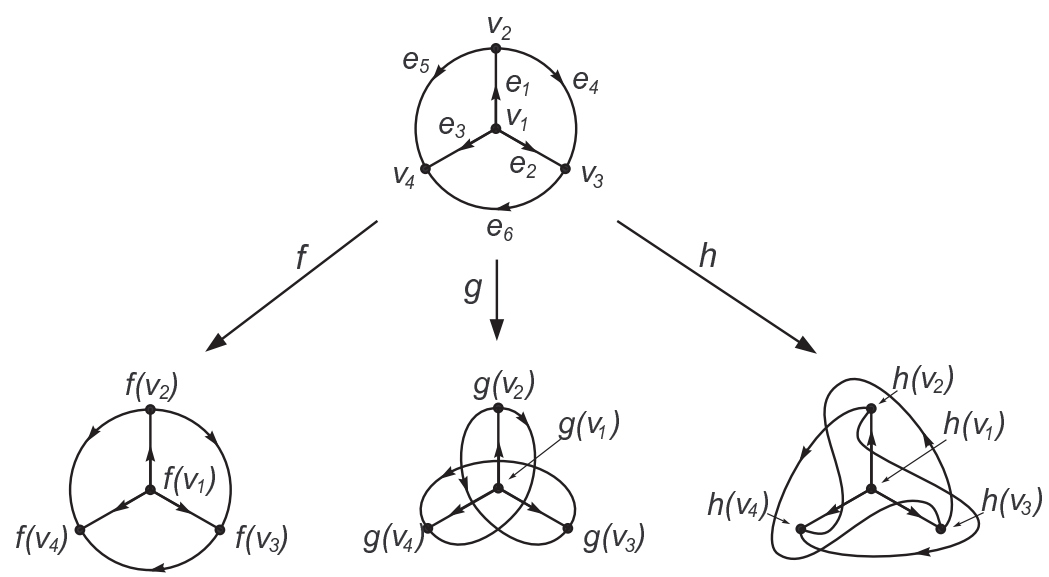

Figure 9.

\section{Proof of Theorem 1.1}

First we show two lemmas which are needed to prove Theorem 1.1.

Lemma 4.1. Each of the local moves as illustrated in Figure 11(4) and (5) are represented by a sequence of moves from the list as illustrated in Figure 1(1), (2), (3) and ambient isotopies.

Proof. See Figures 12 and 13.

We remark here that the local move as illustrated in Figure 11(4) is none other than the Whitney trick. 


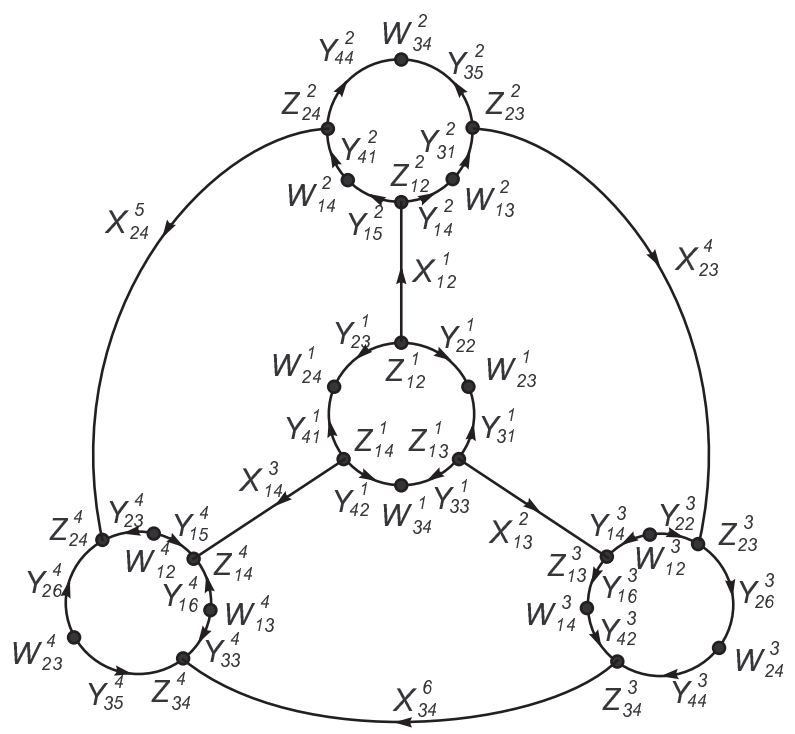

Figure 10.

(4)

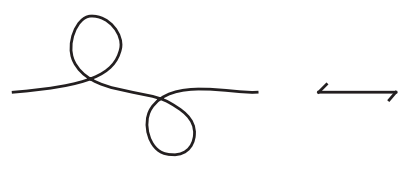

(5)

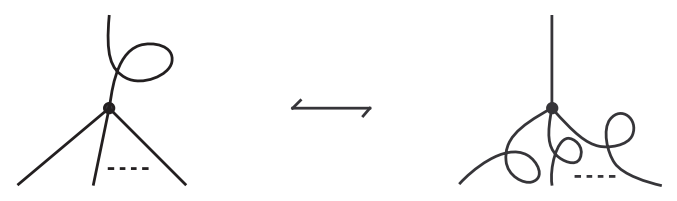

Figure 11. 


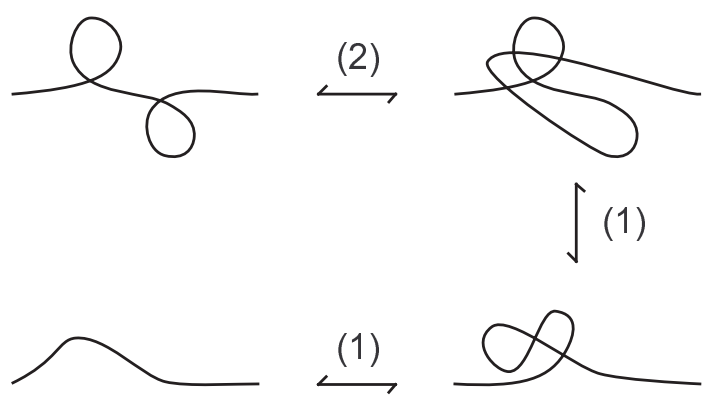

Figure 12.

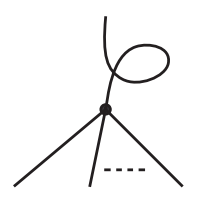

$\stackrel{(3)}{\longleftarrow}$

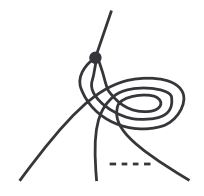

$\int(1),(2)$

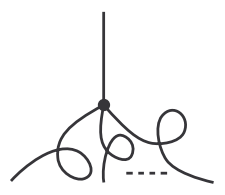

(1), (2)

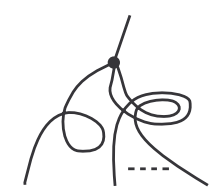

Figure 13. 
Lemma 4.2. Let $G$ be a graph, $H$ a connected subgraph of $G$ and $f$ and $g$ two plane immersions of $G$. If $\mathcal{R}(f)=\mathcal{R}(g)$, then $\mathcal{R}\left(\left.f\right|_{H}\right)=\mathcal{R}\left(\left.g\right|_{H}\right)$.

Proof. Let $i: H \rightarrow G$ be the inclusion. Since $i$ is injective, the homomorphism

$$
i^{*}: H^{1}\left(G^{(0)} ; \mathbb{Z}\right) \longrightarrow H^{1}\left(H^{(0)} ; \mathbb{Z}\right)
$$

is induced by $i$. Clearly $i^{*}(\mathcal{R}(f))=\mathcal{R}\left(\left.f\right|_{H}\right)$ and $i^{*}(\mathcal{R}(g))=\mathcal{R}\left(\left.g\right|_{H}\right)$. Therefore by the assumption we have the desired conclusion.

For a generic plane immersion $f$ and a vertex $v_{s}$ of a graph $G$, a cyclic order of the edges of $G$ incident to $v_{s}$ is determined by considering a neighbourhood $U$ of $v_{s}$ so that $\left.f\right|_{U}$ is an embedding. We call it a cyclic order of $f\left(v_{s}\right)$.

Proof of Theorem 1.1. Since $(1) \Rightarrow(3)$ is shown by Proposition 2.1 and $(2) \Rightarrow(1)$ is clear, it is sufficient to show that $(3) \Rightarrow(2)$. Assume that $\mathcal{R}(f)=\mathcal{R}(g)$. In the following we show that $f$ and $g$ are transformed into each other by the moves as illustrated in Figure 1(1), (2), (3), Figure 11(4), (5) and ambient isotopies. Then by Lemma 4.1, we have the desired conclusion. Since $\mathcal{R}(f)=\mathcal{R}(g)$, by Lemma 4.2 we have that $\mathcal{R}\left(\left.f\right|_{\mathrm{st}\left(v_{s}\right)}\right)=\mathcal{R}\left(\left.g\right|_{\mathrm{st}\left(v_{s}\right)}\right)$ for any vertex $v_{s}$ of $G$. Then by Example 3.12 we have that the cyclic order of $f\left(v_{s}\right)$ is equal to the cyclic order of $g\left(v_{s}\right)$ for any vertex $v_{s}$ of $G$. Let $T_{G}$ be a spanning tree of $G$. By using the moves as illustrated in Figure 1(1), (2), (3), Figure 11(5) and ambient isotopies in case of necessity, we can deform $f$ (resp. $g$ ) so that $\left.f\right|_{T_{G}}$ (resp. $\left.g\right|_{T_{G}}$ ) is an embedding. Since the cyclic order of $f\left(v_{s}\right)$ is equal to the cyclic order of $g\left(v_{s}\right)$ for any vertex $v_{s}$ of $G$, we may assume that $\left.f\right|_{T_{G}}=\left.g\right|_{T_{G}}$. We set $E(G) \backslash E\left(T_{G}\right)=\left\{e_{k_{1}}, e_{k_{2}}, \ldots, e_{k_{\beta}}\right\}$, where $\beta$ denotes the first Betti number of $G$. Let $p_{k_{i}}$ be the unique path on $T_{G}$ which connects the terminal vertices of $e_{k_{i}}$. We denote a cycle $e_{k_{i}} \cup p_{k_{i}}$ by $\gamma_{k_{i}}$. Note that the double points of $\left.f\right|_{\gamma_{k_{i}}}$ (resp. $\left.g\right|_{\gamma_{k_{i}}}$ ) are only the double points of $\left.f\right|_{e_{k_{i}}}$ (resp. $\left.g\right|_{e_{k_{i}}}$ ). Then, by using the moves as illustrated in Figure 1(1), (2), (3), Figure 11(4) and ambient isotopies in case of necessity, we can deform $\left.f\right|_{\gamma_{k_{i}}}$ into the generic plane immersion of $\gamma_{k_{i}}$ as illustrated in Figure 14(1) or $(2)(i=1,2, \ldots, \beta)$. Then, by Lemma 4.2 we have that $\mathcal{R}\left(\left.f\right|_{\gamma_{k_{i}}}\right)=\mathcal{R}\left(\left.g\right|_{\gamma_{k_{i}}}\right)$, namely $\left.f\right|_{\gamma_{k_{i}}}$ and $\left.g\right|_{\gamma_{k_{i}}}$ have the same rotation number. Thus we may assume that $\left.f\right|_{\gamma_{k_{i}}}=\left.g\right|_{\gamma_{k_{i}}}$ for $i=1,2, \ldots, \beta$. This implies that we can deform $f$ and $g$ identically by the moves as illustrated in Figure 1(1), (2), (3), Figure 11(4), (5) and ambient isotopies. This completes the proof.

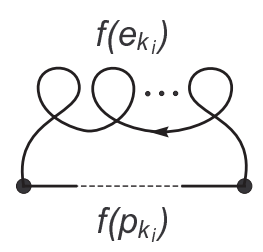

(1)

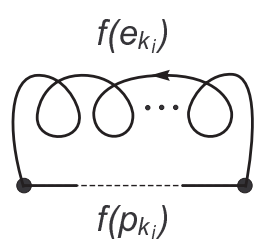

(2)

Figure 14. 


\section{References}

[1] S.T. Hu, Isotopy invariants of topological spaces, Proc. Roy. Soc. London. Ser. A 255 (1960), no. 1282, 331-366.

[2] L.H. Kauffman, Formal knot theory, Mathematical Notes 30, Princeton University Press, Princeton, NJ, 1983.

[3] D.A. Permyakov, Classification of immersions of graphs into a plane, Moscow Univ. Math. Bull. 63 (2008), no. 5, 208-210.

[4] K. Taniyama, Homology classification of spatial embeddings of a graph, Topology Appl. 65 (1995), 205-228.

[5] H. Whitney, On regular closed curves on the plane, Compositio Math. 4 (1937), 276-284.

[6] W.T. Wu, A theory of imbedding, immersion, and isotopy of polytopes in a Euclidean space, Science Press, Peking, 1965.

[7] W.T. Wu, On the isotopy of a complex in a Euclidean space. I, Sci. Sinica 9 (1960), 21-46.

Ryo Nikkuni nick@lab.twcu.ac.jp

Department of Mathematics, School of Arts and Sciences, Tokyo Woman's Christian University, 2-6-1 Zempukuji, Suginami-ku, Tokyo 167-8585, Japan 